\title{
Fratura de complexo zigomaticomaxilar por prática esportiva: relato de caso
}

\author{
Zygomaticomaxillary complex fracture by sports practice: case report \\ Fractura del complejo cigomático-maxilar en la práctica de deportes: \\ reporte de un caso
}

\author{
Gabriel Mulinari dos SANTOS 1 \\ Sara Tiemi Felipe AKABANE2 \\ Erik Neiva Ribeiro de Carvalho REIS1 \\ William Ricardo PIRES ${ }^{1}$ \\ Juliana Zorzi COLÉTE ${ }^{1}$ \\ Francisley Ávila SOUZA ${ }^{3}$ \\ Leonardo Perez FAVERANI ${ }^{3}$ \\ Osvaldo MAGRO FILHO ${ }^{3}$ \\ Idelmo Rangel GARCIA JÚNIOR ${ }^{3}$
}

\author{
1Programa de Pós Graduação em Odontologia, Área de Concentração em Cirurgia e Traumatologia Buco Maxilofacial, \\ Faculdade de Odontologia de Araçatuba, Universidade Estadual Paulista, UNESP, 16015-050, Araçatuba - SP, Brasil \\ ${ }^{2}$ Graduanda em Odontologia, Faculdade de Odontologia de Araçatuba, \\ Universidade Estadual Paulista, UNESP, 16015-050, Araçatuba-SP, Brasil \\ ${ }^{3}$ Disciplina de Cirurgia, Departamento de Cirurgia e Clínica Integrada \\ Faculdade de Odontologia de Araçatuba, Universidade Estadual Paulista, UNESP, 16015-050, Araçatuba - SP, Brasil
}

\begin{abstract}
Resumo
Devido a sua localização projetada na face, o complexo zigomaticomaxilar é uma região comumente acometida por traumas e injúrias, fraturando-se pela ação direta de forças, ocasionando uma mudança em sua projeção anteroposterior e laterolateral. As etiologias dos traumas que podem provocar uma fratura nessa região podem variar, sendo em grande parte as agressões físicas, acidentes de trânsito e esportivos. Objetivo: apresentar um relato de caso clínico de um paciente de 17 anos, gênero masculino, sem histórico de alergias ou comorbidades, vítima de acidente esportivo. Ao exame físico extrabucal foi possível observar edema em hemiface esquerda, degrau palpável em sutura frontozigomática e equimose periorbitária esquerda. Ao exame intrabucal observou-se fratura de pilar zigomático com oclusão mantida. O tratamento cirúrgico foi realizado através da fixação com miniplaca e parafusos do sistema 1.5 em sutura frontozigomática e miniplaca $2.0 \mathrm{em}$ "L" no pilar zigomático. Conclusão: Com base no exposto, a fixação interna rígida com uso de miniplacas em sutura frontozigomática e pilar zigomático mostrou-se efetiva para restabelecer o adequado posicionamento do complexo zigomaticomaxilar, devolvendo também melhores condições funcionais, estéticas e psicológicas ao paciente.
\end{abstract}

Descritores: Fraturas maxilares; Fraturas Zigomáticas; Traumatismos Faciais.

\begin{abstract}
Due to its projected location on the face, the zygomaticomaxillary complex is a region commonly affected by trauma and injuries, fracturing by the direct action of forces, causing a change in its anteroposterior and laterolateral projection. The etiologies of trauma that can cause a fracture in this region can vary, being largely physical aggressions, traffic accidents and sports. Objective: to present a clinical case report of a 17-year-old male patient, with no history of allergies or comorbidities, a victim of a sports accident. On the extra-buccal physical examination it was possible to observe left-sided hemifacial edema, a palpable step-off at the zygomaticofrontal suture and left-side periorbital bruise. Intrabuccal examination showed a zygomatic pillar fracture with maintained occlusion. Surgical treatment was performed by means of miniplate and screws of system 1.5 in zygomaticofrontal suture and mini-plate 2.0 in "L" in the zygomatic pillar. Conclusion: on the basis of the above, the intrafixation with the use of mini-plates in zygomaticofrontal suture and zygomatic pillar was effective in restoring the proper positioning of the zygomaticomaxillary complex, also restoring better functional, aesthetic and psychological conditions to the patient.

Descriptors: Maxillary Fractures; Zygomatic Fractures; Facial Injuries.
\end{abstract}

\section{Resumen}

Debido a su ubicación proyectada en la cara, el complejo cigomático-maxilar es una región comúnmente afectados por traumas y lesiones, fracturando por la acción directa de las fuerzas, provocando un cambio en su proyección anteroposterior y latero-lateral Las etiologías de trauma que puede causar una fractura en esta región pueden variar, siendo abuso, accidentes de tráfico y deportes en gran medida físicos. Objetivo: Presentar un caso clínico de un paciente de 17 años de edad, de sexo masculino, sin antecedentes de alergias o enfermedades concomitantes, víctima de un accidente deportivo. El examen físico extraoral demostró el edema de hemicara izquierda, con un escalafón palpable en la sutura cigomático frontal y equimosis en la región periorbitaria izquierda. En la exploración reveló fractura del complejo cigomático-maxilar y oclusión mantenido. El tratamiento quirúrgico se realizó mediante la fijación con mini placa y tornillos del sistema 1.5 en la sutura cigomática frontal y mini placa 2.0 "L" en el pilar cigomático. Conclusión: La fijación interna rígida con el uso de mini placas en la sutura cigomática frontal y el pilar cigomático fue eficaz para restaurar la posición adecuada del complejo cigomático-maxilar, también volviendo mejores condiciones funcionales, estéticas y psicológicas del paciente.

Descriptores: Fracturas Maxilares; Fracturas Cigomáticas; Traumatismos Faciales.

\section{INTRODUÇÃO}

Em virtude de sua localização e projeção na face, o complexo zigomático é um sítio comumente acometido por traumas e injúrias faciais. Tal estrutura é constituída pelo osso zigomático e arco zigomático, sendo este uma estrutura óssea de anatomia simples, linear, formado pela prolongação óssea do osso zigomático e osso temporal. O arco zigomático pode fraturar-se pela ação de traumas de pequena intensidade, devido a sua posição e estrutura frági ${ }^{1-5}$. 
O osso zigomático possui quatro processos: frontal, temporal, orbital e maxilar. Ele atua através da dissipação e transmissão de forças mastigatórias, principalmente através do pilar zigomático, oferecendo também proteção ao globo ocular 6 .

O complexo zigomático possui relação com ossos, cavidades e tecidos faciais, sendo eles: maxila, temporal, frontal, seio maxilar, esfenoide, parede lateral e assoalho de órbita, fissura orbital superior e inferior, nervo infraorbitário, processo coronoide, músculos temporal, masseter, zigomático maior e menor, reto inferior, órbita, dentre outros ${ }^{6}$.

O complexo zigomaticomaxilar é uma estrutura que exerce papel importante para a função e estética facial. Tal complexo funciona como uma barreira óssea, separando os elementos orbitários dos seios maxilares e fossas temporais ${ }^{7}$. $\mathrm{Na}$ literatura, diversos autores apontam que o complexo zigomático maxilar ocupa o terceiro lugar dentre as fraturas faciais ${ }^{8}$.

Com relação à etiologia de tais fraturas, podemos citar em maior número os acidentes automobilísticos, as agressões físicas e acidentes esportivos ${ }^{9}$.

Diante de uma fratura, o diagnóstico realizado pelo cirurgião bucomaxilofacial deve obter informações acerca da história do trauma (tipo, intensidade e tempo decorrido), realizar o exame físico e solicitar exames de imagem, fundamentais para o diagnóstico e tratamento.

Os exames comumente realizados incluem a radiografia em posição de Waters e de Hirtz e tomografia computadorizada, a qual representa um grande avanço e facilita a localização precisa das fraturas, visto que não há sobreposição de imagens como nas radiografias ${ }^{10}$.

Dentre os principais sinais e sintomas que ocorrem, podemos citar: degrau palpável ao longo da pele em região infraorbitária, assimetria facial devido ao afundamento da região zigomática, parestesia do nervo infraorbitário, diplopia, edema e hematoma palpebral, equimose subconjuntival, epistaxe, degrau no pilar zigomático, edema e equimose em mucosa jugal ${ }^{10}$.

Dentre as classificações propostas, em 1961 Knight e North ${ }^{11}$ consagraram as fraturas do complexo zigomático através do grau de cominução e deslocamento apresentados na radiografia de Waters (Tabela 1 ).

Tabela 1: Classificação das fraturas do complexo zigomático, segundo Knight e North ${ }^{11}$

\begin{tabular}{ll}
\hline GRUPO & DESCRIÇÃO \\
\hline I & Sem deslocamento do zigoma \\
II & Fraturas de arco zigomático \\
III & Com deslocamento, sem redução \\
IV & Com deslocamento e rotação medial \\
V & Com deslocamento e rotação lateral \\
VI & Complexas \\
\hline
\end{tabular}

Em 2003 Manganello-Souza et al. classificaram as fraturas em 3 tipos: zigomáticas (tipo I ou II) e orbitozigomática (tipo III). As fraturas do tipo I apresentam pequeno deslocamento do osso, fratura não cominutiva e ausência de disfunção ocular. Tipo II: grande deslocamento do osso, fratura cominutiva sem necessidade de reconstrução e presença de disfunção ocular. Tipo III: apresenta grande deslocamento do osso, fratura cominutiva com necessidade de reconstrução, presença de disfunção ocular, fratura no corpo do zigoma e necessidade de fixar o arco zigomático ${ }^{12}$.

Muito se discute acerca do tipo de tratamento para tais fraturas, sendo o tempo decorrido entre o acidente e o atendimento pelo cirurgião bucomaxilofacial, de grande importância para sua indicação.
Os tratamentos de fraturas faciais mostram avanços no protocolo. Onde inicialmente se realizava o bloqueio maxilomandibular ou utilização de fio de aço, evoluiu-se até os dias atuais, para a utilização em larga escala da fixação interna rígida (FIR). Esta constitui um meio seguro, estável e eficaz de tratamento, diminuindo o índice de complicações pós-operatórias e possibilitando o retorno mais rápido do paciente às suas funções ${ }^{13}$.

\section{CASO CLÍNICO}

Paciente do gênero masculino, 17 anos, leucoderma, sem histórico de alergias ou comorbidades, vítima de acidente esportivo, foi encaminhado a Santa Casa de Misericórdia de Araçatuba suspeita de trauma facial. Durante o exame clínico, o paciente relatou ter recebido um chute frontal enquanto praticava karatê, referindo que "sentia seu rosto afundado", o mesmo apresentava visão turva e diplopia. Ao exame físico extraoral foi possível observar edema em hemi-face esquerda, degrau palpável em sutura frontozigomática, hemorragia subconjuntival e equimose periorbitária esquerda (Figura 1). Os movimentos oculares se mostravam preservados e sem complicações oftalmológicas. Ao exame intraoral observou-se degrau do pilar zigomático, corroborado pela tomografia (Figura 2), porém com a oclusão mantida.

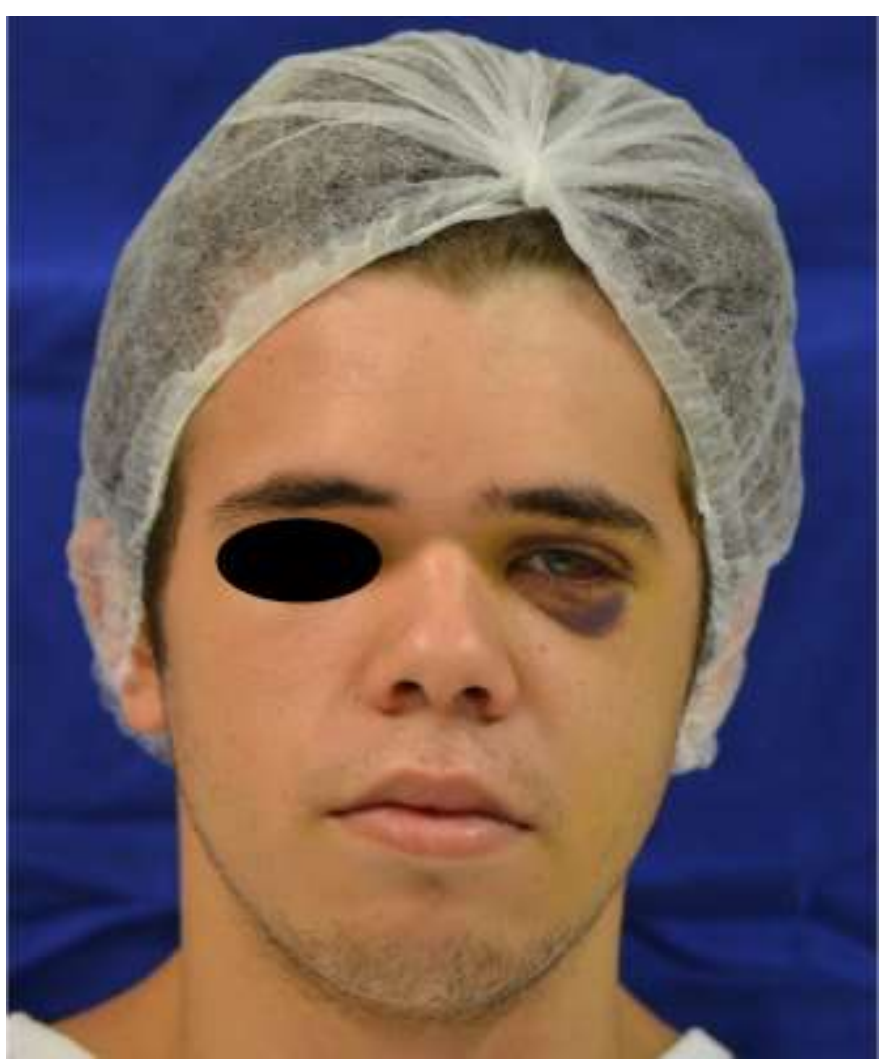

Figura 1: Pré-operatório. Evidenciando hemorragia subconjuntival e equimose periorbitária esquerda e assimetria facial com perda da projeção do complexo zigomaticomaxilar esquerdo.

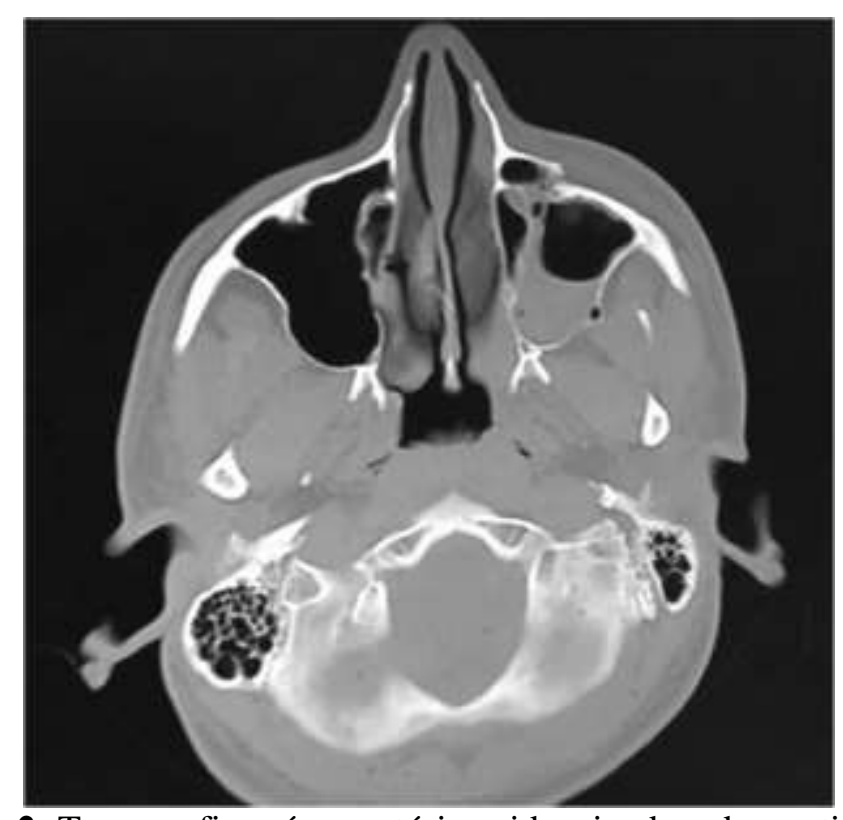

Figura 2: Tomografia pré-operatória evidenciando a descontinuidade do contorno do complexo zigomatíco provocado pela fratura. 
O tratamento cirúrgico do paciente se deu sob anestesia geral, onde após a antissepsia intra e extra-oral, montagem dos campos esteréis e anestesia local com lidocaína (epinefrina 1:100.000), foi realizado o acesso cirúrgico em fundo de sulco vestibular superior (Figura 3) e acesso supraciliar do lado esquerdo (Figura 4).

Foi realizada a redução e fixação com mini-placas e parafusos do sistema $1.5 \mathrm{em}$ sutura fronto-zigomática (Figura 5) e mini-placa 2.0 em "L" no pilar zigomático (Figura 6).

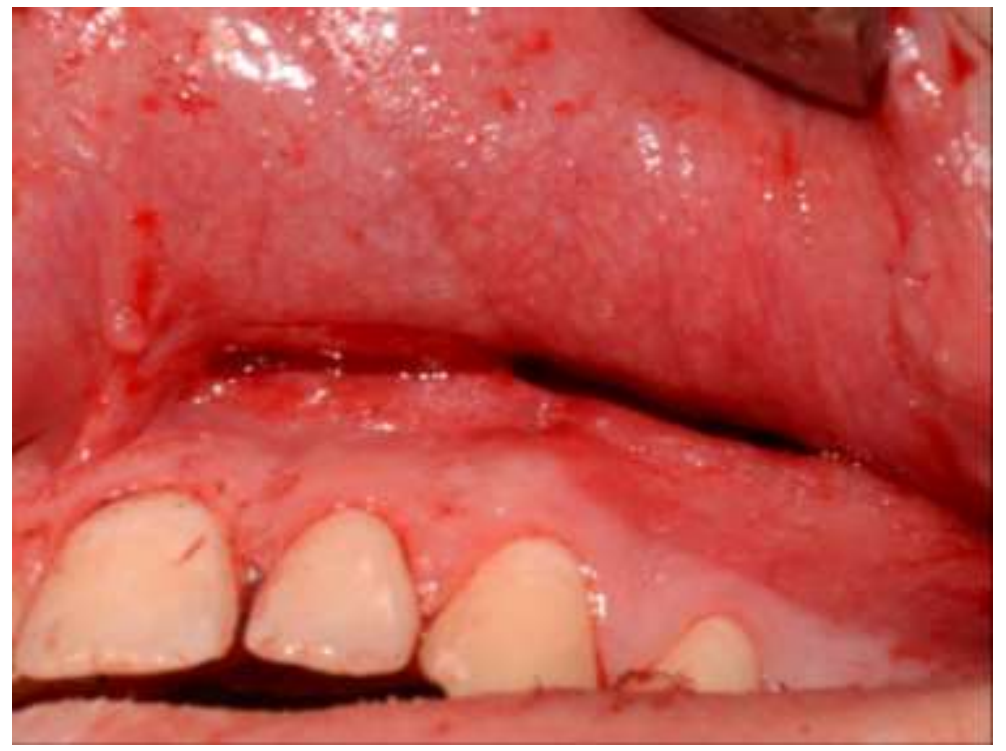

Figura 3: Acesso em fundo de sulco vestibular.

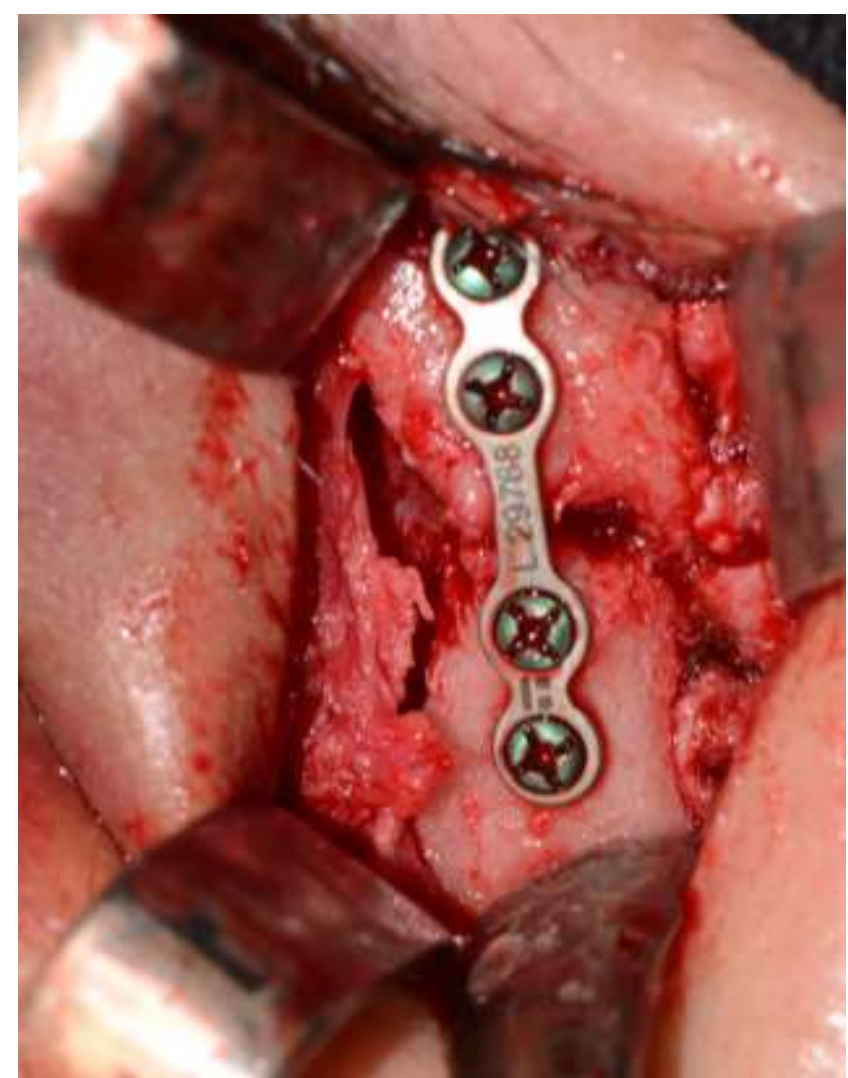

Figura 5: Fixação com miniplacas 1.5 em sutura frontozigomática.

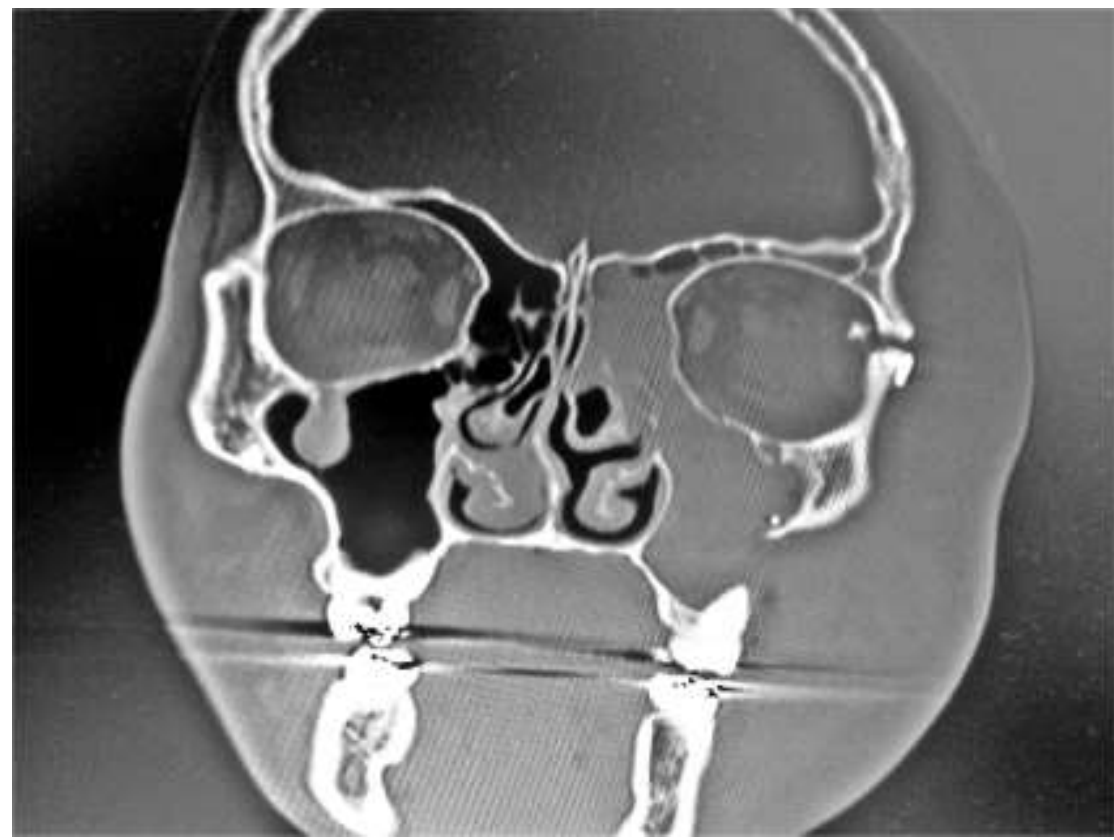

Figura 7: Tomografia pós-operatória em corte coronal mostrando redução da fratura em sutura frontozigomática.
O pilar canino permaneceu integro. A síntese tecidual foi realizada por planos utilizando poliglactina-910 4.0 violeta nos tecidos profundos, e superficialmente em pele com nylon 5.0. A imagem tomográfica pós-operatório já mostrou correta redução e fixação das fraturas, e o posicionamento mais adequado de todo complexo zigomático-maxilar no corte coronal (Figura 7) e corte axial (Figura 8)

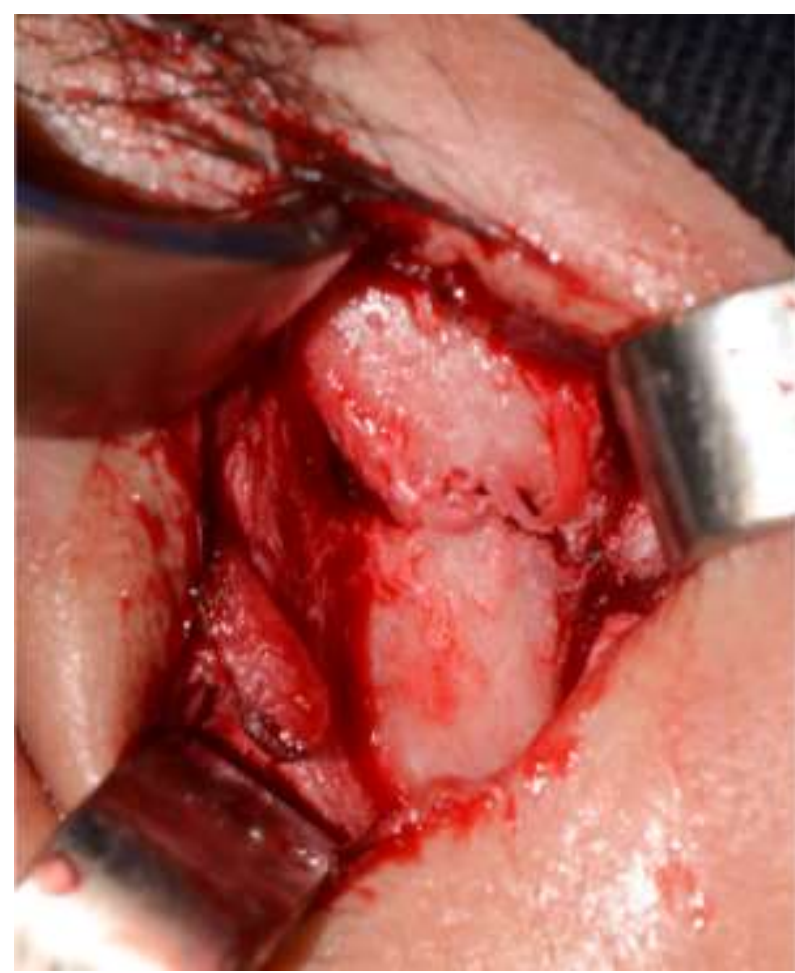

Figura 4: Acesso supraciliar e fratura em sutura frontozigomática.

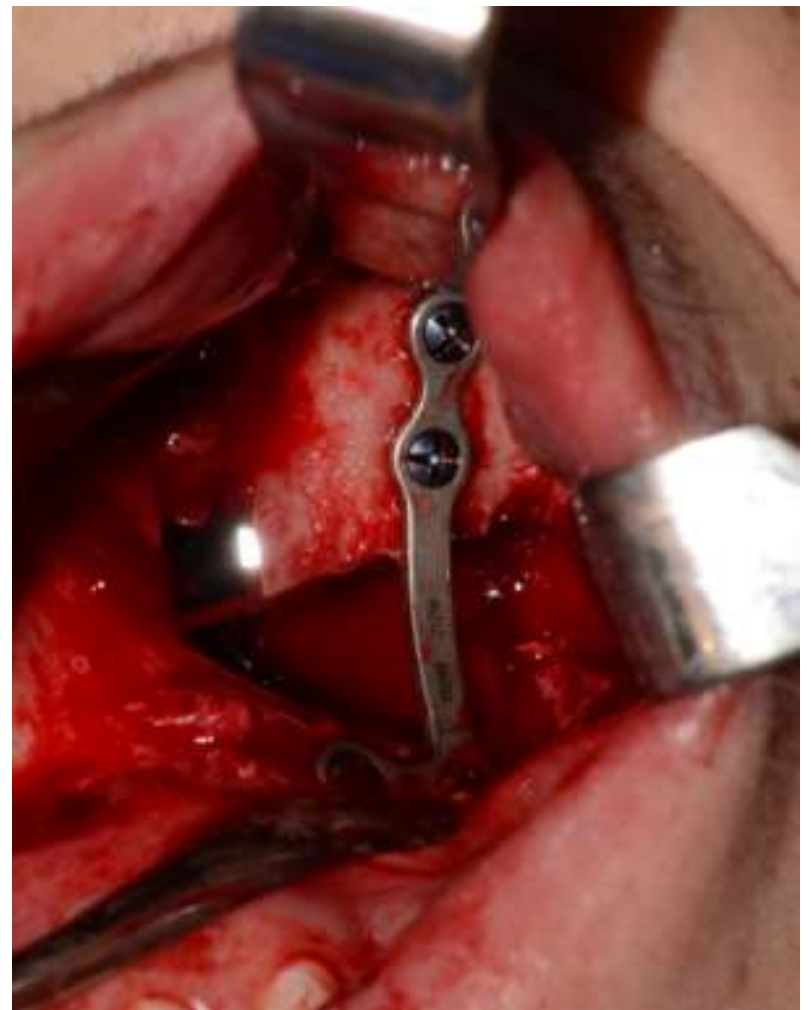

Figura 6: Fixação com miniplacas 2.0 em pilar zigomático

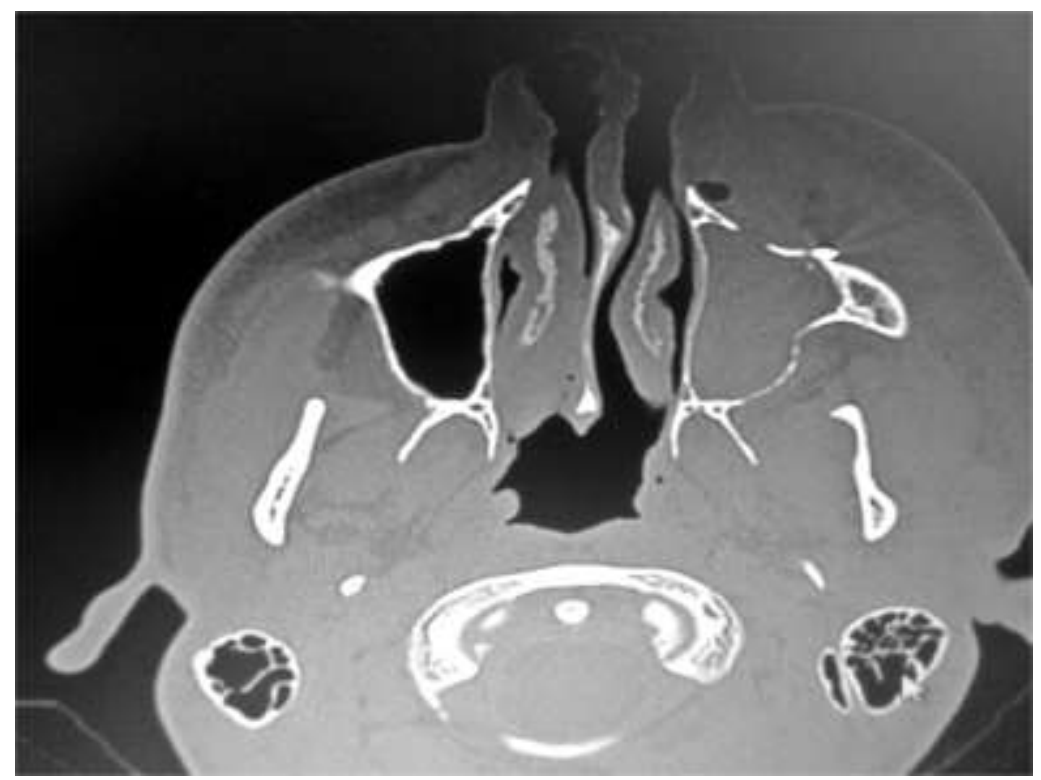

Figura 8: Tomografia pós-operatória em corte axial mostrando correta redução da fratura pelo contorno do complexo zigomático. 
A Figura 9 mostra o aspecto pós-operatório imediato do paciente, sem sinais e sintomas e infecção, com edema compatível ao procedimento cirúrgico realizado. O pósoperatório de 15 dias mostra bom aspecto estético após a redução da fratura, devolvendo a projeção do complexo zigomático-maxilar e corrigindo a assimetria facial (Figura 10).

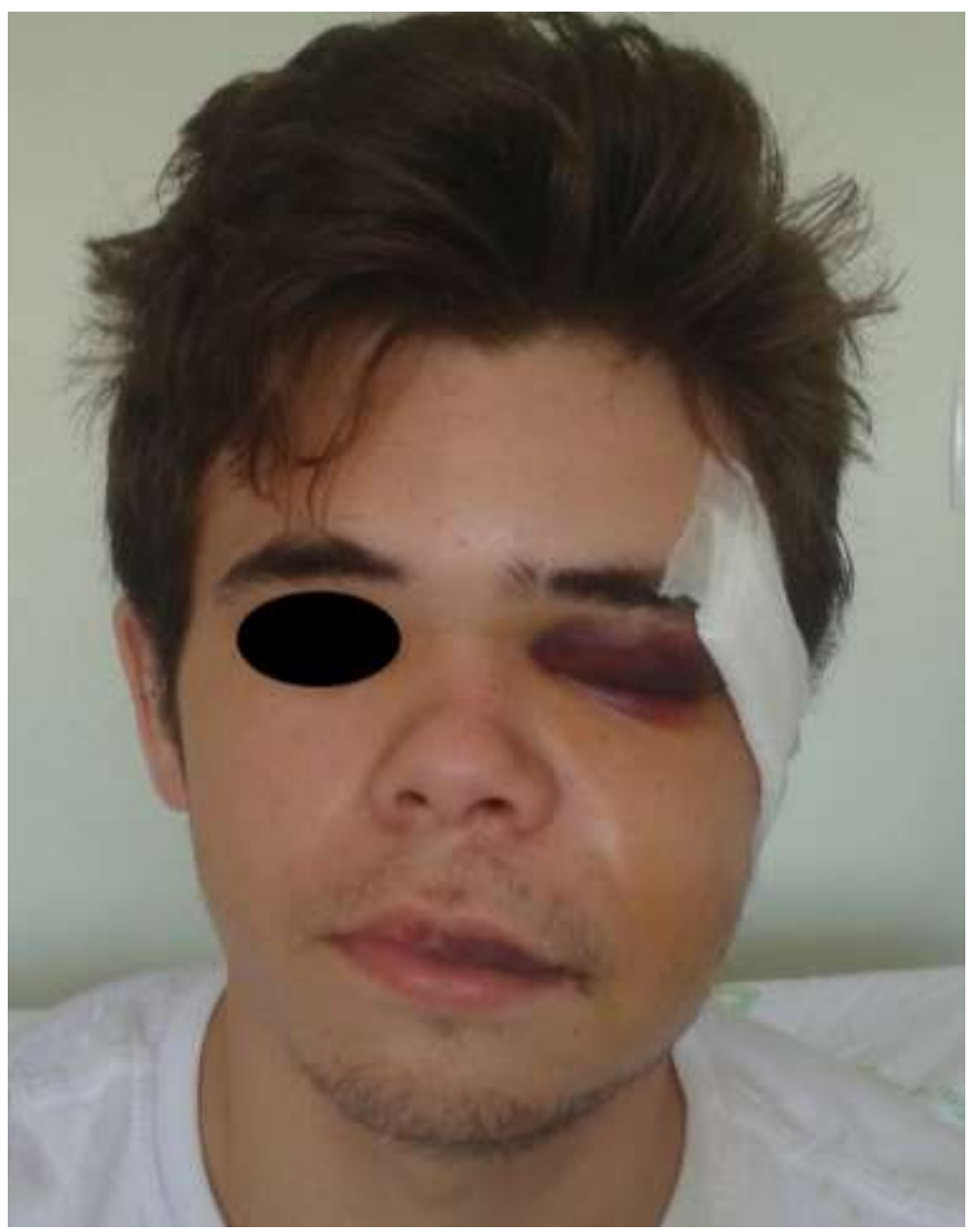

Figura 9: Aspecto pós-operatório imediato do paciente, sem sinais e sintomas e infecção, com edema compatível ao procedimento cirúrgico realizado.

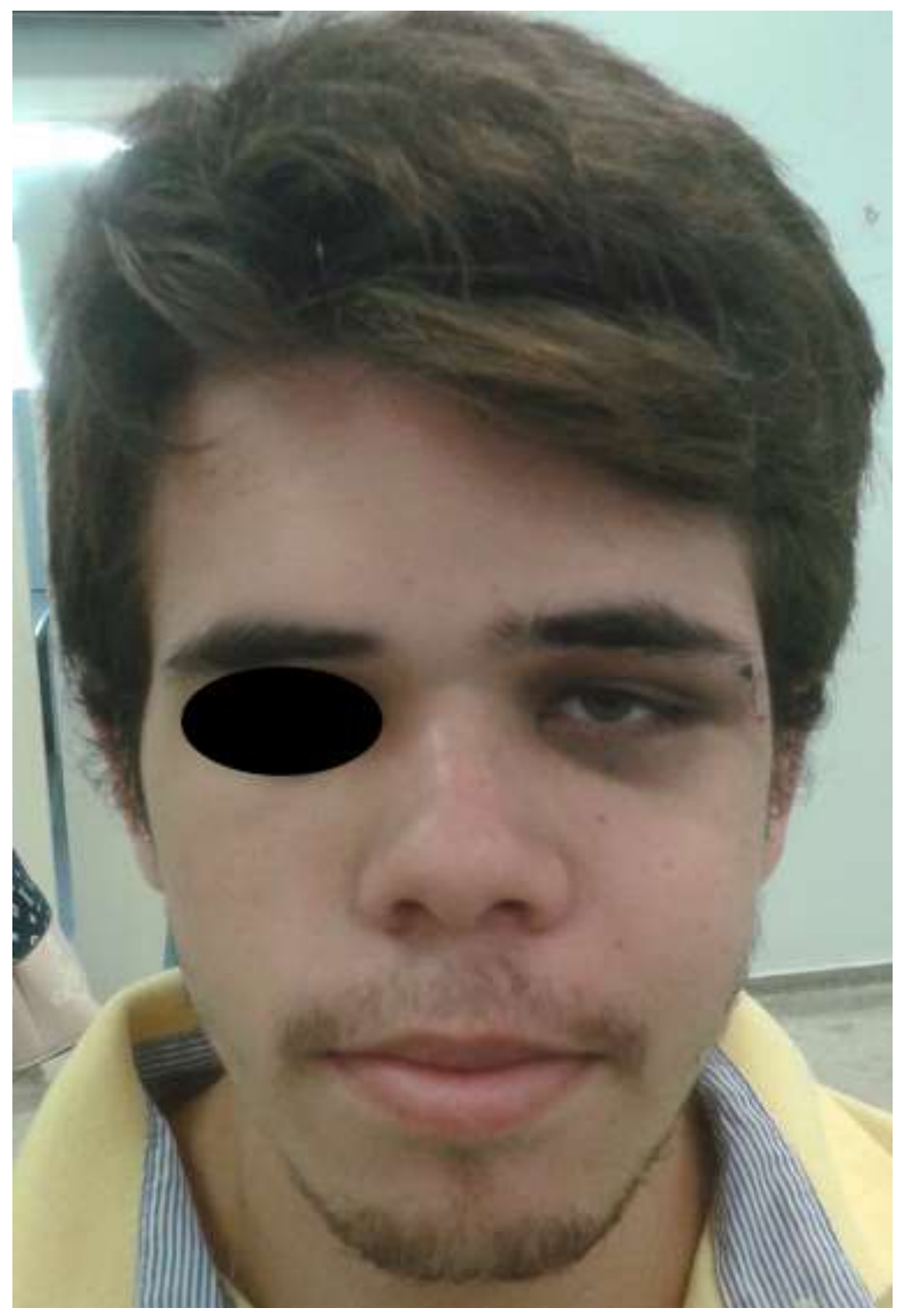

Figura 10: Pós-operatório de 15 dias, onde é possível notar o bom aspecto estético após a redução da fratura, devolvendo a projeção do complexo zigomaticomaxilar e corrigindo a assimetria facial.

\section{DISCUSSÃO}

A opção de abordar uma fratura do zigoma com redução aberta ou fechada ainda é controversa. No caso exposto, o tratamento proposto foi realizado com redução aberta para melhor redução e estabilização da fratura do grupo IV segundo classificação de Knight e North de $1961^{11}$. A fixação interna rígida, que consiste em uma fixação através da utilização de placas e parafusos em fraturas do complexo zigomático podem ser feitas em pilar zigomático, sutura frontozigomática, pilares caninos e margem infraorbitária.

Autores citam a fixação em três pontos sendo indicada quando a fratura se encontra instável ${ }^{14}$. Optamos por realizar a osteossíntese com placas e parafusos de fixação interna rígida em dois pontos (pilar frontozigomático, e pilar zigomaticomaxilar). Visto que o terceiro ponto sendo considerado o pilar canino permaneceu com sua integridade óssea mantida, e com isso, não houve necessidade de realizar sua fixação.

O tratamento cirúrgico das fraturas do complexo zigomático e arco zigomático estão indicados quando há presença de fraturas com deslocamentos significativos, associados a limitação da abertura bucal, comprometimento da motilidade e funções oculares, assim como defeitos estéticos na face que geram assimetria facial, como queixa no presente caso ${ }^{15}$.

Por meio do tratamento cirúrgico proposto foi possível notar o correto reposicionamento do zigomático, podendo ser observado o bom aspecto pós-operatório imediato do paciente, sem sinais e sintomas e infecção, e com edema compatível ao do procedimento cirúrgico realizado.

\section{CONCLUSÃO}

Com base no exposto e visto que o pilar canino estava mantido, não havendo necessidade de sua fixação, podemos concluir que a fixação interna rígida com uso de miniplacas em sutura frontozigomática e pilar zigomático mostrou-se efetiva para restabelecer o adequado posicionamento e projeção do complexo zigomático-maxilar. Ademais, o tratamento cirúrgico realizado conferiu melhores condições funcionais, estéticas e psicológicas ao jovem paciente.

\section{REFERÊNCIAS}

1. Dodson TB, Perrot DH, Kaban LB, Gordon NC. Fixation of mandibular fractures : a comparative study of rigid internal fixation and standard fixation techiques. J Oral Maxillofac Surg. 1990; 48(4):362-6.

2. Adi M, Ogden GR, Chisholm DM. An analysis of mandibular fractures in Dundee, Scotland (1977 to 1985). Br J Oral Maxillofac Surg. 1990; 28(3):194- 9.

3. Peterson LJ, Ellis E, Hupp JR. Cirurgia Oral e Maxilofacial Contemporânea. $3^{\mathrm{a}}$ ed. Rio de Janeiro: Guanabara Koogan; 2000. p. 487-511.

4. Miloro M, Ghali GE, Larsen PE, Waite PD. Princípios de cirurgia bucomaxilofacial de Peterson. $2^{a}$ ed. São Paulo: Santos; 2008. p. 435- 462.

5. Hammer B. Fraturas Orbitárias - Diagnóstico, tratamento cirúrgico, correções secundarias. São Paulo: Santos; 2005.

6. Rizzolo RJC, Madeira MC. Anatomia Facial com fundamentos de Anatomia Geral. 3.ed. Sarvier; 2009

7. Monnazzi MS, Hochuli-Vieira E, Gabrielli MAC, Gabrielli MFR, Pereira-Filho VA. Avaliação de complicações tardias em fraturas maxilares do tipo Le Fort. Rev Fac Odontol Bauru. 2002; 10(4):257- 62. 
8. Vetter JD, Topazian RG, Goldeberg MH, Smith DG. Facial fractures occuring in a medium-sized metropolitan area: recent trends. Int J Oral Maxillofac Surg. 1991; 20(4):214-6.

9. Falcão MFL, Leite-Segundo AV, Silveira MMF. Estudo epidemiológico de 1758 fraturas faciais tratadas no hospital da restauração, Recife/PE. Rev cir traumatol buco-maxilo-fac. 2005; 5(3):65-72

10. Dingman RO, Natvig P. Cirurgia das fraturas faciais. 3 ed. São Paulo: Santos; 2004.

11. Knight JS, North JF. The classification of malar fractures: an analysis of displacement as a guide to treatment. Br J Plast Surg. 1961; 13:325-39.

12. Manganello LCS, Soares CR, Santos LCB. Fraturas do malar: diagnóstico e tratamento. Rev Ass Paul Cirur Dent. 1982; 36:100

13. Klotch DW, Gilliland R. Internal fixation Vs conventional therapy in midface fractures. J Trauma. 1987; 27(10):1136-45

14. Kelley P, Hopper R, Gruss J. Evaluation and treatment of zygomatic fractures. Plast Reconstr Surg. 2007; 120(7 Suppl 2):5S-15S

15. Hollier LH, Thornton J, Pazmino P, Stal S. The management of orbitozygomatic fractures. Plast Reconstr Surg. 2003; 111(7):2386-92.

\section{CONFLITO DE INTERESSES}

Os autores declaram não haver conflitos de interesse.

\section{AUTOR PARA CORRESPONDÊNCIA}

Gabriel Mulinari dos Santos

gabriel_mulinari@hotmail.com

Submetido em 19/01/2017 Aceito em 26/02/2017 